\title{
Word Clustering and Disambiguation Based on Co-occurrence Data
}

\author{
Hang Li and Naoki Abe \\ Theory NEC Laboratory, Real World Computing Partnership \\ c/o C\&C Media Research Laboratories., NEC \\ 4-1-1 Miyazaki, Miyamae-ku, Kawasaki 216-8555, Japan \\ \{lihang,abe\}@ccm.cl.nec.co.jp
}

\begin{abstract}
We address the problem of clustering words (or constructing a thesaurus) based on co-occurrence data, and using the acquired word classes to improve the accuracy of syntactic disambiguation. We view this problem as that of estimating a joint probability distribution specifying the joint probabilities of word pairs, such as noun verb pairs. We propose an efficient algorithm based on the Minimum Description Length (MDL) principle for estimating such a probability distribution. Our method is a natural extension of those proposed in (Brown et al., 1992) and ( $\mathrm{Li}$ and $\mathrm{Abe}, 1996)$, and overcomes their drawbacks while retaining their advantages. We then combined this clustering method with the disambiguation method of ( $\mathrm{Li}$ and Abe, 1995) to derive a disambiguation method that makes use of both automatically constructed thesauruses and a hand-made thesaurus. The overall disambiguation accuracy achieved by our method is $85.2 \%$, which compares favorably against the accuracy (82.4\%) obtained by the state-of-the-art disambiguation method of (Brill and Resnik, 1994).
\end{abstract}

\section{Introduction}

We address the problem of clustering words, or that of constructing a thesaurus, based on co-occurrence data. We view this problem as that of estimating a joint probability distribution over word pairs, specifying the joint probabilities of word pairs, such as noun verb pairs. In this paper, we assume that the joint distribution can be expressed in the following manner, which is stated for noun verb pairs for the sake of readability: The joint probability of a noun and a verb is expressed as the product of the joint probability of the noun class and the verb class which the noun and the verb respectively belong to, and the conditional probabilities of the noun and the verb given their respective classes.

As a method for estimating such a probability distribution, we propose an algorithm based on the Minimum Description Length (MDL) principle. Our clustering algorithm iteratively merges noun classes and verb classes in turn, in a bottom up fashion. For each merge it performs, it calculates the increase in data description length resulting from merging any noun (or verb) class pair, and performs the merge having the least increase in data description length, provided that the increase in data description length is less than the reduction in model description length.

There have been a number of methods proposed in the literature to address the word clustering problem (e.g., (Brown et al., 1992; Pereira et al., 1993; Li and Abe, 1996)). The method proposed in this paper is a natural extension of both $\mathrm{Li} \&$ Abe's and Brown et al's methods, and is an attempt to overcome their drawbacks while retaining their advantages.

The method of Brown et al, which is based on the Maximum Likelihood Estimation (MLE), performs a merge which would result in the least reduction in (average) mutual information. Our method turns out to be equivalent to performing the merge with the least reduction in mutual information, provided that the reduction is below a certain threshold which depends on the size of the co-occurrence data and the number of classes in the current situation. This method, based on the MDL principle, takes into account both the fit to data and the simplicity of a model, and thus can help cope with the over-fitting problem that the MLE-based method of Brown et al faces.

The model employed in ( $\mathrm{Li}$ and Abe, 1996) is based on the assumption that the word distribution within a class is a uniform distribution, i.e. every word in a same class is generated with an equal probability. Employing such a model has the undesirable tendency of classifying into different classes those words that have similar co-occurrence patterns but have different absolute frequencies. The proposed method, in contrast, employs a model in which different words within a same class can have different conditional generation probabilities, and thus can classify words in a way that is not affected by words' absolute frequencies and resolve the problem faced by the method of (Li and Abe, 1996).

We evaluate our clustering method by using the word classes and the joint probabilities obtained by 
it in syntactic disambiguation experiments. Our experimental results indicate that using the word classes constructed by our method gives better disambiguation results than when using $\mathrm{Li} \& \mathrm{Abe}$ or Brown et al's methods. By combining thesauruses automatically constructed by our method and an existing hand-made thesaurus (WordNet), we were able to achieve the overall accuracy of $85.2 \%$ for ppattachment disambiguation, which compares favorably against the accuracy $(82.4 \%)$ obtained using the state-of-the-art method of (Brill and Resnik, 1994).

\section{Probability Model}

Suppose available to us are co-occurrence data over two sets of words, such as the sample of verbs and the head words of their direct objects given in Fig. 1. Our goal is to (hierarchically) cluster the two sets of words so that words having similar co-occurrence patterns are classified in the same class, and output a thesaurus for each set of words.

wine
beer
bread
rice $\begin{array}{ccc}0 & 3 & 1 \\ 0 & 5 & 1 \\ 4 & 0 & 2 \\ 4 & 0 & 0\end{array}$

Figure 1: Example co-occurrence data

We can view this problem as that of estimating the best probability model from among a class of models of (probability distributions) which can give rise to the co-occurrence data.

In this paper, we consider the following type of probability models. Assume without loss of generality that the two sets of words are a set of nouns $\mathcal{N}$ and a set of verbs $\mathcal{V}$. A partition $T_{n}$ of $\mathcal{N}$ is a set of noun-classes satisfying $\cup_{C_{n} \in T_{n}} C_{n}=\mathcal{N}$ and $\forall C_{i}, C_{j} \in T_{n}, C_{i} \cap C_{j}=\emptyset$. A partition $T_{v}$ of $\mathcal{V}$ can be defined analogously. We then define a probability model of noun-verb co-occurrence by defining the joint probability of a noun $n$ and a verb $v$ as the product of the joint probability of the noun and verb classes that $n$ and $v$ belong to, and the conditional probabilities of $n$ and $v$ given their classes, that is,

$$
P(n, v)=P\left(C_{n}, C_{v}\right) \cdot P\left(n \mid C_{n}\right) \cdot P\left(v \mid C_{v}\right)
$$

where $C_{n}$ and $C_{v}$ denote the (unique) classes to which $n$ and $v$ belong. In this paper, we refer to this model as the 'hard clustering model,' since it is based on a type of clustering in which each word can belong to only one class. Fig. 2 shows an example of the hard clustering model that can give rise to the co-occurrence data in Fig. 1.

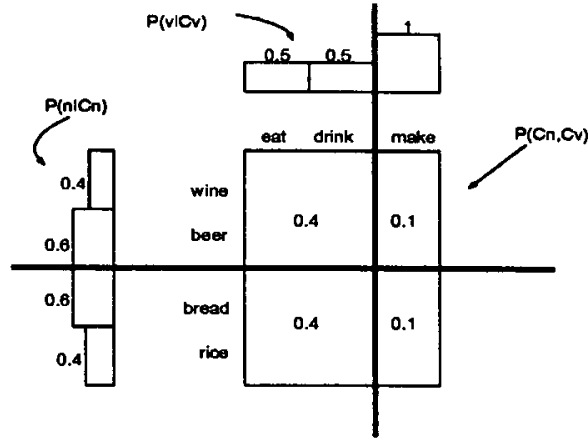

Figure 2: Example hard clustering model

\section{Parameter Estimation}

A particular choice of partitions for a hard clustering model is referred to as a 'discrete' hard-clustering model, with the probability parameters left to be estimated. The values of these parameters can be estimated based on the co-occurrence data by the Maximum Likelihood Estimation. For a given set of co-occurrence data

$$
\mathcal{S}=\left\{\left(n_{1}, v_{1}\right),\left(n_{2}, v_{2}\right), \ldots,\left(n_{m}, v_{m}\right)\right\}
$$

the maximum likelihood estimates of the parameters are defined as the values that maximize the following likelihood function with respect to the data:

$\prod_{i=1}^{m} P\left(n_{i}, v_{i}\right)=\prod_{i=1}^{m}\left(P\left(n_{i} \mid C_{n_{i}}\right) \cdot P\left(v_{i} \mid C_{v_{i}}\right) \cdot P\left(C_{n_{i}}, C_{v_{i}}\right)\right)$.

It is easy to see that this is possible by setting the parameters as

$$
\begin{gathered}
\hat{P}\left(C_{n}, C_{v}\right)=\frac{f\left(C_{n}, C_{v}\right)}{m}, \\
\forall x \in \mathcal{N} \cup \mathcal{V}, \hat{P}\left(x \mid C_{x}\right)=\frac{f(x)}{f\left(C_{x}\right)}
\end{gathered}
$$

Here, $m$ denotes the entire data size, $f\left(C_{n}, C_{v}\right)$ the frequency of word pairs in class pair $\left(C_{n}, C_{v}\right), f(x)$ the frequency of word $x$, and $f\left(C_{x}\right)$ the frequency of words in class $C_{x}$.

\section{Model Selection Criterion}

The question now is what criterion should we employ to select the best model from among the possible models. Here we adopt the Minimum Description Length (MDL) principle. MDL (Rissanen, 1989) is a criterion for data compression and statistical estimation proposed in information theory.

In applying MDL, we calculate the code length for encoding each model, referred to as the 'model description length' $L(M)$, the code length for encoding 
the given data through the model, referred to as the 'data description length' $L(\mathcal{S} \mid M)$ and their sum:

$$
L(M, \mathcal{S})=L(M)+L(\mathcal{S} \mid M) .
$$

The MDL principle stipulates that, for both data compression and statistical estimation, the best probability model with respect to given data is that which requires the least total description length.

The data description length is calculated as

$$
L(\mathcal{S} \mid M)=-\sum_{(n, v) \in \mathcal{S}} \log \hat{P}(n, v),
$$

where $\hat{P}$ stands for the maximum likelihood estimate of $P$ (as defined in Section 3).

We then calculate the model description length as

$$
L(M)=\frac{k}{2} \log m,
$$

where $k$ denotes the number of free parameters in the model, and $m$ the entire data size. ${ }^{1}$ In this paper, we ignore the code length for encoding a 'discrete model,' assuming implicitly that they are equal for all models and consider only the description length for encoding the parameters of a model as the model description length.

If computation time were of no concern, we could in principle calculate the total description length for each model and select the optimal model in terms of MDL. Since the number of hard clustering models is of order $O\left(N^{N} \cdot V^{V}\right)$, where $N$ and $V$ denote the size of the noun set and the verb set, respectively, it would be infeasible to do so. We therefore need to devise an efficient algorithm that heuristically performs this task.

\section{Clustering Algorithm}

The proposed algorithm, which we call '2DClustering,' iteratively selects a suboptimal MDLmodel from among those hard clustering models which can be obtained from the current model by merging a noun (or verb) class pair. As it turns out, the minimum description length criterion can be reformalized in terms of (average) mutual information, and a greedy heuristic algorithm can be formulated to calculate, in each iteration, the reduction of mutual information which would result from merging any noun (or verb) class pair, and perform the merge

\footnotetext{
${ }^{1}$ We note that there are alternative ways of calculating the parameter description length. For example, we can separately encode the different types of probability parameters; the joint probabilities $P\left(C_{n}, C_{v}\right)$, and the conditional probabilities $P\left(n \mid C_{n}\right)$ and $P\left(v \mid C_{v}\right)$. Since these alternatives are approximations of one another asymptotically, here we use only the simplest formulation. In the full paper, we plan to compare the empirical behavior of the alternatives.
}

having the least mutual information reduction, provided that the reduction is below a variable threshold.

2D-Clustering $\left(\mathcal{S}, b_{n}, b_{v}\right)$

( $\mathcal{S}$ is the input co-occurrence data, and $b_{n}$ and $b_{v}$ are positive integers.)

1. Initialize the set of noun classes $T_{n}$ and the set of verb classes $T_{v}$ as:

$$
T_{n}=\{\{n\} \mid n \in \mathcal{N}\}, T_{v}=\{\{v\} \mid v \in \mathcal{V}\},
$$

where $\mathcal{N}$ and $\mathcal{V}$ denote the noun set and the verb set, respectively.

2. Repeat the following three steps:

(a) execute $\operatorname{Merge}\left(\mathcal{S}, T_{n}, T_{v}, b_{n}\right)$ to update $T_{n}$,

(b) execute Merge $\left(\mathcal{S}, T_{v}, T_{n}, b_{v}\right)$ to update $T_{v}$,

(c) if $T_{n}$ and $T_{v}$ are unchanged, go to Step 3 .

3. Construct and output a thesaurus for nouns based on the history of $T_{n}$, and one for verbs based on the history of $T_{v}$.

Next, we describe the procedure of 'Merge,' as it is being applied to the set of noun classes with the set of verb classes fixed.

$\operatorname{Merge}\left(\mathcal{S}, T_{n}, T_{v}, b_{n}\right)$

1. For each class pair in $T_{n}$, calculate the reduction of mutual information which would result from merging them. (The details will follow.) Discard those class pairs whose mutual information reduction (2) is not less than the threshold of

$$
\frac{\left(k_{B}-k_{A}\right) \cdot \log m}{2 \cdot m}
$$

where $m$ denotes the total data size, $k_{B}$ the number of free parameters in the model before the merge, and $k_{A}$ the number of free parameters in the model after the merge. Sort the remaining class pairs in ascending order with respect to mutual information reduction.

2. Merge the first $b_{n}$ class pairs in the sorted list.

3. Output current $T_{n}$.

We perform (maximum of) $b_{n}$ merges at step 2 for improving efficiency, which will result in outputting an at-most $b_{n}$-ary tree. Note that, strictly speaking, once we perform one merge, the model will change and there will no longer be a guarantee that the remaining merges still remain justifiable from the viewpoint of MDL.

Next, we explain why the criterion in terms of description length can be reformalized in terms of mutual information. We denote the model before a merge as $M_{B}$ and the model after the merge as 
$M_{A}$. According to MDL, $M_{A}$ should have the least increase in data description length

$$
\delta L_{d a t}=L\left(\mathcal{S} \mid M_{A}\right)-L\left(\mathcal{S} \mid M_{B}\right)>0,
$$

and at the same time satisfies

$$
\delta L_{d a t}<\frac{\left(k_{B}-k_{A}\right) \log m}{2} .
$$

This is due to the fact that the decrease in model description length equals

$$
L\left(M_{B}\right)-L\left(M_{A}\right)=\frac{\left(k_{B}-k_{A}\right) \log m}{2}>0,
$$

and is identical for each merge.

In addition, suppose that $M_{A}$ is obtained by merging two noun classes $C_{i}$ and $C_{j}$ in $M_{B}$ to a single noun class $C_{i j}$. We in fact need only calculate the difference between description lengths with respect to these classes, i.e.,

$$
\begin{aligned}
\delta L_{d a t}= & -\sum_{C_{v} \in T_{v}} \sum_{n \in C_{i j}, v \in C_{v}} \log \hat{P}(n, v) \\
& +\sum_{C_{v} \in T_{v}} \sum_{n \in C_{i}, v \in C_{v}} \log \hat{P}(n, v) \\
& +\sum_{C_{v} \in T_{v}} \sum_{n \in C_{j}, v \in C_{v}} \log \hat{P}(n, v) .
\end{aligned}
$$

Now using the identity

$$
\begin{aligned}
P(n, v) & =\frac{P(n)}{P\left(C_{n}\right)} \cdot \frac{P(v)}{P\left(C_{v}\right)} \cdot P\left(C_{n}, C_{v}\right) \\
& =\frac{P\left(C_{n}, C_{v}\right)}{P\left(C_{n}\right) \cdot P\left(C_{v}\right)} \cdot P(n) \cdot P(v)
\end{aligned}
$$

we can rewrite the above as

$$
\begin{aligned}
& \delta L_{d a t}=-\sum_{C_{v} \in T_{v}} f\left(C_{i j}, C_{v}\right) \log \frac{\hat{P}\left(C_{i j}, C_{v}\right)}{\hat{P}\left(C_{i j}\right) \cdot \hat{P}\left(C_{v}\right)} \\
& +\sum_{C_{v} \in T_{v}} f\left(C_{i}, C_{v}\right) \log \frac{\hat{P}\left(C_{i}, C_{v}\right)}{\hat{P}\left(C_{i}\right) \cdot \hat{P}\left(C_{v}\right)} \\
& +\sum_{C_{v} \in T_{v}} f\left(C_{j}, C_{v}\right) \log \frac{\hat{P}\left(C_{j}, C_{v}\right)}{\hat{P}\left(C_{j}\right) \cdot \hat{P}\left(C_{v}\right)} .
\end{aligned}
$$

Thus, the quantity $\delta L_{d a t}$ is equivalent to the mutual information reduction times the data size. ${ }^{2}$ We conclude therefore that in our present context, a clustering with the least data description length increase is equivalent to that with the least mutual information decrease.

Canceling out $\hat{P}\left(C_{v}\right)$ and replacing the probabilities with their maximum likelihood estimates, we obtain

$$
\begin{aligned}
& \frac{1}{m} \delta L_{d a t}=\frac{1}{m}\left(-\sum_{C_{v} \in T_{v}}\left(f\left(C_{i}, C_{v}\right)+f\left(C_{j}, C_{v}\right)\right)\right. \\
& \log \frac{f\left(C_{i}, C_{v}\right)+f\left(C_{j}, C_{v}\right)}{f\left(C_{i}\right)+f\left(C_{j}\right)} \\
& +\sum_{C_{v} \in T_{v}} f\left(C_{i}, C_{v}\right) \log \frac{f\left(C_{i}, C_{v}\right)}{f\left(C_{i}\right)} \\
& \left.+\sum_{C_{v} \in T_{v}} f\left(C_{j}, C_{v}\right) \log \frac{f\left(C_{j}, C_{v}\right)}{f\left(C_{j}\right)}\right) .
\end{aligned}
$$

\footnotetext{
${ }^{2}$ Average mutual information between $T_{n}$ and $T_{v}$ is defined as$$
I\left(T_{n}, T_{v}\right)=\sum_{C_{n} \in T_{n}} \sum_{C_{v} \in T_{v}}\left(P\left(C_{n}, C_{v}\right) \log \frac{P\left(C_{n}, C_{v}\right)}{P\left(C_{n}\right) \cdot P\left(C_{v}\right)}\right) .
$$

Therefore, we need calculate only this quantity for each possible merge at Step 1 of Merge.

In our implementation of the algorithm, we first load the co-occurrence data into a matrix, with nouns corresponding to rows, verbs to columns. When merging a noun class in row $i$ and that in row $j(i<j)$, for each $C_{v}$ we add $f\left(C_{i}, C_{v}\right)$ and $f\left(C_{j}, C_{v}\right)$ obtaining $f\left(C_{i j}, C_{v}\right)$, write $f\left(C_{i j}, C_{v}\right)$ on row $i$, move $f\left(C_{\text {last }}, C_{v}\right)$ to row $j$, and reduce the matrix by one row.

By the above implementation, the worst case time complexity of the algorithm is $O\left(N^{3} \cdot V+V^{3} \cdot N\right)$ where $N$ denotes the size of the noun set, $V$ that of the verb set. If we can merge $b_{n}$ and $b_{v}$ classes at each step, the algorithm will become slightly more efficient with the time complexity of $O\left(\frac{N^{3}}{b_{n}} \cdot V+\frac{V^{3}}{b_{v}}\right.$. $N)$.

\section{Related Work}

\subsection{Models}

We can restrict the hard clustering model (1) by assuming that words within a same class are generated with an equal probability, obtaining

$$
P(n, v)=P\left(C_{n}, C_{v}\right) \cdot \frac{1}{\left|C_{n}\right|} \cdot \frac{1}{\left|C_{v}\right|},
$$

which is equivalent to the model proposed by ( $\mathrm{Li}$ and Abe, 1996). Employing this restricted model has the undesirable tendency to classify into different classes those words that have similar co-occurrence patterns but have different absolute frequencies.

The hard clustering model defined in (1) can also be considered to be an extension of the model proposed by Brown et al. First, dividing (1) by $P(v)$, we obtain

$$
\frac{P(n, v)}{P(v)}=P\left(C_{n} \mid C_{v}\right) \cdot P\left(n \mid C_{n}\right) \cdot\left(\frac{P\left(C_{v}\right) \cdot P\left(v \mid C_{v}\right)}{P(v)}\right),
$$

Since hard clustering implies $\frac{P\left(C_{v}\right) \cdot P\left(v \mid C_{v}\right)}{P(v)}=1$ holds, we have

$$
P(n \mid v)=P\left(C_{n} \mid C_{v}\right) \cdot P\left(n \mid C_{n}\right) .
$$

In this way, the hard clustering model turns out to be a class-based bigram model and is similar to Brown et al's model. The difference is that the model of (3) assumes that the clustering for $C_{n}$ and the clustering for $C_{v}$ can be different, while the model of Brown et al assumes that they are the same.

A very general model of noun verb joint probabilities is a model of the following form:

$P(n, v)=\sum_{C_{n} \in \Gamma_{n}} \sum_{C_{v} \in \Gamma_{v}} P\left(C_{n}, C_{v}\right) \cdot P\left(n \mid C_{n}\right) \cdot P\left(v \mid C_{v}\right)$. 
Here $\Gamma_{n}$ denotes a set of noun classes satisfying $\cup_{C_{n} \in \Gamma_{n}} C_{n}=\mathcal{N}$, but not necessarily disjoint. Similarly $\Gamma_{v}$ is a set of not necessarily disjoint verb classes. We can view the problem of clustering words in general as estimation of such a model. This type of clustering in which a word can belong to several different classes is generally referred to as 'soft clustering.' If we assume in the above model that each verb forms a verb class by itself, then (4) becomes

$$
P(n, v)=\sum_{C_{n} \in \Gamma_{n}} P\left(C_{n}, v\right) \cdot P\left(n \mid C_{n}\right)
$$

which is equivalent to the model of Pereira et al. On the other hand, if we restrict the general model of (4) so that both noun classes and verb classes are disjoint, then we obtain the hard clustering model we propose here (1). All of these models, therefore, are some special cases of (4). Each specialization comes with its merit and demerit. For example, employing a model of soft clustering will make the clustering process more flexible but also make the learning process more computationally demanding. Our choice of hard clustering obviously has the merits and demerits of the soft clustering model reversed.

\subsection{Estimation criteria}

Our method is also an extension of that proposed by Brown et al from the viewpoint of estimation criterion. Their method merges word classes so that the reduction in mutual information, or equivalently the increase in data description length, is minimized. Their method has the tendency to overfit the training data, since it is based on MLE. Employing MDL can help solve this problem.

\section{Disambiguation Method}

We apply the acquired word classes, or more specifically the probability model of co-occurrence, to the problem of structural disambiguation. In particular, we consider the problem of resolving pp-attachment ambiguities in quadruples, like (see, girl, with, telescope) and that of resolving ambiguities in compound noun triples, like (data, base, system). In the former, we determine to which of 'see' or 'girl' the phrase 'with telescope' should be attached. In the latter, we judge to which of 'base' or 'system' the word 'data' should be attached.

We can perform pp-attachment disambiguation by comparing the probabilities

$$
\hat{P}_{\text {with }} \text { (telescope|see), } \hat{P}_{\text {with }} \text { (telescopelgirl). }
$$

If the former is larger, we attach 'with telescope' to 'see;' if the latter is larger we attach it to 'girl;' otherwise we make no decision. (Disambiguation on compound noun triples can be performed similarly.)
Since the number of probabilities to be estimated is extremely large, estimating all of these probabilities accurately is generally infeasible (i.e., the data sparseness problem). Using our clustering model to calculate these conditional probabilities (by normalizing the joint probabilities with marginal probabilities) can solve this problem.

We further enhance our disambiguation method by the following back-off procedure: We first estimate the two probabilities in question using hard clustering models constructed by our method. We also estimate the probabilities using an existing (hand-made) thesaurus with the 'tree cut' estimation method of ( $\mathrm{Li}$ and Abe, 1995), and use these probability values when the probabilities estimated based on hard clustering models are both zero. Finally, if both of them are still zero, we make a default decision.

\section{Experimental Results}

\subsection{Qualitative evaluation}

In this experiment, we used heuristic rules to extract verbs and the head words of their direct objects from the tagged texts of the WSJ corpus (ACL/DCI CDROM1) consisting of 126,084 sentences.

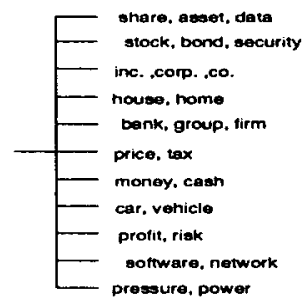

Figure 3: A part of a constructed thesaurus

We then constructed a number of thesauruses based on these data, using our method. Fig. 3 shows a part of a thesaurus for 100 randomly selected nouns, based on their appearances as direct objects of 20 randomly selected verbs. The thesaurus seems to agree with human intuition to some degree, although it is constructed based on a relatively small amount of co-occurrence data. For example, 'stock,' 'security,' and 'bond' are classified together, despite the fact that their absolute frequencies in the data vary a great deal $(272,59$, and 79 , respectively.) The results demonstrate a desirable feature of our method, namely, it classifies words based solely on the similarities in co-occurrence data, and is not affected by the absolute frequencies of the words.

\subsection{Compound noun disambiguation}

We extracted compound noun doubles (e.g., 'data base') from the tagged texts of the WSJ corpus and used them as training data, and then conducted 
structural disambiguation on compound noun triples (e.g., 'data base system').

We first randomly selected 1,000 nouns from the corpus, and extracted compound noun doubles containing those nouns as training data and compound noun triples containing those nouns as test data. There were 8,604 training data and 299 test data. We hand-labeled the test data with the correct disambiguation 'answers.'

We performed clustering on the nouns on the left position and the nouns on the right position in the training data by using both our method ('2DClustering') and Brown et al's method ('Brown'). We actually implemented an extended version of their method, which separately conducts clustering for nouns on the left and those on the right (which should only improve the performance).

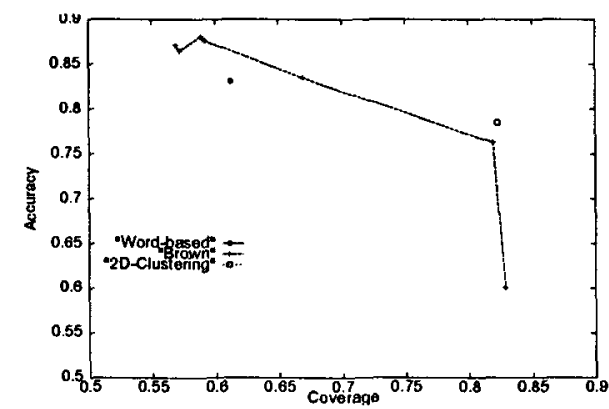

Figure 4: Compound noun disambiguation results

We next conducted structural disambiguation on the test data, using the probabilities estimated based on 2D-Clustering and Brown. We also tested the method of using the probabilities estimated based on word co-occurrences, denoted as 'Word-based.' Fig. 4 shows the results in terms of accuracy and coverage, where coverage refers to the percentage of test data for which the disambiguation method was able to make a decision. Since for Brown the number of classes finally created has to be designed in advance, we tried a number of alternatives and obtained results for each of them. (Note that, for 2D-Clustering, the optimal number of classes is automatically selected.)

Table 1: Compound noun disambiguation results

\begin{tabular}{|lc|}
\hline Method & Acc.(\%) \\
\hline Default & 59.2 \\
Word-based + Default & 73.9 \\
Brown + Default & 77.3 \\
2D-Clustering + Default & 78.3 \\
\hline
\end{tabular}

Tab. 1 shows the final results of all of the above methods combined with 'Default,' in which we at- tach the first noun to the neighboring noun when a decision cannot be made by each of the methods. We see that 2D-Clustering+Default performs the best. These results demonstrate a desirable aspect of 2D-Clustering, namely, its ability of automatically selecting the most appropriate level of clustering, resulting in neither over-generalization nor under-generalization.

\subsection{PP-attachment disambiguation}

We extracted triples (e.g., 'see, with, telescope') from the bracketed data of the WSJ corpus (Penn Tree Bank), and conducted PP-attachment disambiguation on quadruples. We randomly generated ten sets of data consisting of different training and test data and conducted experiments through 'tenfold cross validation,' i.e., all of the experimental results reported below were obtained by taking average over ten trials.

Table 2: PP-attachment disambiguation results

\begin{tabular}{|lcc|}
\hline Method & Cov.(\%) & Acc.(\%) \\
\hline Default & 100 & 56.2 \\
Word-based & 32.3 & 95.6 \\
Brown & 51.3 & 98.3 \\
2D-Clustering & 51.3 & 98.3 \\
Li-Abe96 & 37.3 & 94.7 \\
WordNet & 74.3 & 94.5 \\
NounClass-2DC & 42.6 & 97.1 \\
\hline
\end{tabular}

We constructed word classes using our method ('2D-Clustering') and the method of Brown et al ('Brown'). For both methods, following the proposal due to (Tokunaga et al., 1995), we separately conducted clustering with respect to each of the 10 most frequently occurring prepositions (e.g., 'for,' 'with,' etc). We did not cluster words for rarely occurring prepositions. We then performed disambiguation based on 2D-Clustering and Brown. We also tested the method of using the probabilities estimated based on word co-occurrences, denoted as 'Word-based.'

Next, rather than using the conditional probabilities estimated by our method, we only used the noun thesauruses constructed by our method, and applied the method of ( $\mathrm{Li}$ and Abe, 1995) to estimate the best 'tree cut models' within the thesauruses ${ }^{3}$ in order to estimate the conditional probabilities like those in (5). We call the disambiguation method using these probability values 'NounClass-2DC.' We also tried the analogous method using thesauruses constructed by the method of ( $\mathrm{Li}$ and Abe, 1996)

\footnotetext{
${ }^{3}$ The method of ( $\mathrm{Li}$ and Abe, 1995) outputs a 'tree cut model' in a given thesaurus with conditional probabilities attached to all the nodes in the tree cut. They use MDL to select the best tree cut model.
} 
and estimating the best tree cut models (this is exactly the disambiguation method proposed in that paper). Finally, we tried using a hand-made thesaurus, WordNet (this is the same as the disambiguation method used in ( $\mathrm{Li}$ and Abe, 1995)). We denote these methods as 'Li-Abe96' and 'WordNet,' respectively.

Tab. 2 shows the results for all these methods in terms of coverage and accuracy.

Table 3: PP-attachment disambiguation results

\begin{tabular}{|lc|}
\hline Method & Acc.(\%) \\
\hline Word-based + Default & 69.5 \\
Brown + Default & 76.2 \\
2D-Clustering + Default & 76.2 \\
Li-Abe96 + Default & 71.0 \\
WordNet + Default & 82.2 \\
NounClass-2DC + Default & 73.8 \\
\hline \hline 2D-Clustering + WordNet + Default & 85.2 \\
Brill-Resnik & 82.4 \\
\hline
\end{tabular}

We then enhanced each of these methods by using a default rule when a decision cannot be made, which is indicated as '+Default.' Tab. 3 shows the results of these experiments.

We can make a number of observations from these results. (1) 2D-Clustering achieves a broader coverage than NounClass-2DC. This is because in order to estimate the probabilities for disambiguation, the former exploits more information than the latter. (2) For Brown, we show here only its best result, which happens to be the same as the result for 2DClustering, but in order to obtain this result we had to take the trouble of conducting a number of tests to find the best level of clustering. For 2D-Clustering, this was done once and automatically. Compared with $\mathrm{Li}$-Abe96, 2D-Clustering clearly performs better. Therefore we conclude that our method improves these previous clustering methods in one way or another. (3) 2D-Clustering outperforms WordNet in term of accuracy, but not in terms of coverage. This seems reasonable, since an automatically constructed thesaurus is more domain dependent and therefore captures the domain dependent features better, and thus can help achieve higher accuracy. On the other hand, with the relatively small size of training data we had available, its coverage is smaller than that of a general purpose hand made thesaurus. The result indicates that it makes sense to combine automatically constructed thesauruses and a handmade thesaurus, as we have proposed in Section 7.

This method of combining both types of thesauruses '2D-Clustering+WordNet+Default' was then tested. We see that this method performs the best. (See Tab. 3.) Finally, for comparison, we tested the 'transformation-based error-driven learning' proposed in (Brill and Resnik, 1994), which is a state-of-the-art method for pp-attachment disambiguation. Tab. 3 shows the result for this method as 'Brill-Resnik.' We see that our disambiguation method also performs better than Brill-Resnik. (Note further that for Brill \& Resnik's method, we need to use quadruples as training data, whereas ours only requires triples.)

\section{Conclusions}

We have proposed a new method of clustering words based on co-occurrence data. Our method employs a probability model which naturally represents cooccurrence patterns over word pairs, and makes use of an efficient estimation algorithm based on the MDL principle. Our clustering method improves upon the previous methods proposed by Brown et al and ( $\mathrm{Li}$ and $\mathrm{Abe}, 1996$ ), and furthermore it can be used to derive a disambiguation method with overall disambiguation accuracy of $85.2 \%$, which improves the performance of a state-of-the-art disambiguation method.

The proposed algorithm, 2D-Clustering, can be used in practice, as long as the data size is at the level of the current Penn Tree Bank. Yet it is still relatively computationally demanding, and thus an important future task is to further improve on its computational efficiency.

\section{Acknowledgement}

We are grateful to Dr. S. Doi of NEC C\&C Media Res. Labs. for his encouragement. We thank Ms. Y. Yamaguchi of NIS for her programming efforts.

\section{References}

E. Brill and P. Resnik. A rule-based approach to prepositional phrase attachment disambiguation. Proc. of COLING'94, pp. 1198-1204.

P. F. Brown, V. J. Della Pietra, P. V. deSouza, J. C. Lai, and R. L. Mercer. 1992. Class-based ngram models of natural language. Comp. Ling., $18(4): 283-298$.

H. Li and N. Abe. 1995. Generalizing case frames using a thesaurus and the MDL principle. Comp. Ling., (to appear).

H. Li and N. Abe. 1996. Clustering words with the MDL principle. Proc. of COLING'96, pp. 4-9.

F. Pereira, N. Tishby, and L. Lee. 1993. Distributional clustering of English words. Proc. of ACL'93, pp. 183-190.

J. Rissanen. 1989. Stochastic Complexity in Statistical Inquiry. World Scientific Publishing Co., Singapore.

T. Tokunaga, M. Iwayama, and H. Tanaka. Automatic thesaurus construction based-on grammatical relations. Proc. of IJCAI'95, pp. 1308-1313. 